University of Wollongong

Research Online

Faculty of Engineering and Information

Faculty of Engineering and Information

Sciences - Papers: Part A

Sciences

$1-1-2014$

Computational analysis of compressive strain hardening exponents of bimetal with pearlitic steel and low carbon steel

Xingjian Gao

University of Wollongong, xg306@uowmail.edu.au

Zhengyi Jiang

University of Wollongong, jiang@uow.edu.au

Dongbin Wei

University of Technology Sydney, dwei@uow.edu.au

Sihai Jiao

Baosteel, Baosteel Research Institute

Jingtao Han

University of Science and Technology of China

Follow this and additional works at: https://ro.uow.edu.au/eispapers

Part of the Engineering Commons, and the Science and Technology Studies Commons

Research Online is the open access institutional repository for the University of Wollongong. For further information contact the UOW Library: research-pubs@uow.edu.au 


\title{
Computational analysis of compressive strain hardening exponents of bimetal with pearlitic steel and low carbon steel
}

\begin{abstract}
The compressive strain hardening behaviour of a novel bimetal with pearlitic steel and low carbon steel was investigated by computational analysis based on the isothermal compression tests in a wide range of deformation temperature and strain rate. The Hollomon's equation was employed to calculate the strain hardening exponent (SHE) with the assistance of mathematical manipulation. The result shows that the logarithmic relationship between the flow stress and plastic strain of the bimetal is highly nonlinear, which results in the variation of the SHE of the bimetal. This variation reflects the dynamic competition between the strain hardening and softening mechanism by the varying value of the SHE in the range of 0.4 to-0.4. Furthermore, the influences of deformation temperature and strain rate on the SHE are significant. With decreasing temperature and increasing strain rate, the strain hardening of the bimetal was enhanced, while the dynamic recrystallisation was activated under the opposite conditions with the evidence of negative SHE value.
\end{abstract}

\section{Keywords}

computational, exponents, hardening, bimetal, pearlitic, steel, low, carbon, compressive, strain, analysis

\section{Disciplines}

Engineering | Science and Technology Studies

\section{Publication Details}

Gao, X., Jiang, Z., Wei, D., Jiao, S. \& Han, J. (2014). Computational analysis of compressive strain hardening exponents of bimetal with pearlitic steel and low carbon steel. Applied Mechanics and Materials, 553 71-75. 


\title{
Computational analysis of compressive strain hardening exponents of bimetal with pearlitic steel and low carbon steel
}

\author{
Xingjian $\mathrm{Gao}^{1, \mathrm{a}}$, Zhengyi Jiang ${ }^{1, \mathrm{~b},{ }^{*}}$, Dongbin $\mathrm{We}^{1,{ }^{1,2} \mathrm{c}}$, Sihai Jiao ${ }^{3, \mathrm{e}}$, Jingtao \\ $\operatorname{Han}^{4, f}$ \\ ${ }^{1}$ School of Mechanical, Materials and Mechatronic Engineering, University of Wollongong, \\ Wollongong NSW 2522, Australia \\ ${ }^{2}$ School of Electrical, Mechanical and Mechatronic Systems, University of Technology, Sydney NSW \\ 2007, Australia \\ ${ }^{3}$ Baosteel Research Institute, Baoshan Iron \& Steel Co., Ltd., Shanghai 200941, China \\ ${ }^{4}$ School of Materials Science and Engineering, University of Science \& Technology Beijing, Beijing \\ 100083, China \\ axy306@uowmail.edu.au, biang@uow.edu.au, cdwei@uow.edu.au, eshjiao@baosteel.com, \\ fhanjt@ustb.edu.cn, ${ }^{*}$ Corresponding author
}

Keywords: Strain-hardening exponent, Bimetal, Hot compression, Dynamic recrystallisation.

\begin{abstract}
The compressive strain hardening behaviour of a novel bimetal with pearlitic steel and low carbon steel was investigated by computational analysis based on the isothermal compression tests in a wide range of deformation temperature and strain rate. The Hollomon's equation was employed to calculate the strain hardening exponent (SHE) with the assistance of mathematical manipulation. The result shows that the logarithmic relationship between the flow stress and plastic strain of the bimetal is highly non-linear, which results in the variation of the SHE of the bimetal. This variation reflects the dynamic competition between the strain hardening and softening mechanism by the varying value of the SHE in the range of 0.4 to -0.4. Furthermore, the influences of deformation temperature and strain rate on the SHE are significant. With decreasing temperature and increasing strain rate, the strain hardening of the bimetal was enhanced, while the dynamic recrystallisation was activated under the opposite conditions with the evidence of negative SHE value.
\end{abstract}

\section{Introduction}

Recently, a novel bimetal consisting of pearlitic steel (PS) and low carbon steel (LCS) was fabricated to integrate attractive strength / ductility balance, moderate hardness with good wear resistance and superior weldability into a single product [1]. In order to improve mechanical properties of the bimetal, various thermomechanical treatments such as hot rolling and forging processes are generally performed on the bimetallic billet. However, a new challenge was encountered during hot-working process because of different deformation responses on different metallic layers. Although previous research [2,3] has developed the processing map and constitutive model of the bimetal, the strain hardening behaviour during hot deformation was not discussed.

Strain hardening is a natural reflection of dislocation multiplication and interaction due to plastic deformation $[4,5]$. It is of major importance in forming operations since it not only increases the strengths of the material, but also competes against dynamic softening behaviours (including dynamic recovery and dynamic recrystallisation) with an increase of plastic strain. The strain hardening exponent (SHE), which is the indicator that signifies the strain hardening characteristic of a material, was proposed in the uniform plastic deformation stage during the tensile process [6]. Conventionally, the value of the SHE lies between 0 and 1 before necking occurred. So it is a material constant that used to describe the maximum amount of uniform strain in tensile deformation. Whereas, the range of the SHE can be extended $[7,8]$ in an isothermal compression test because there is no necking 
phenomenon. The variation of the SHE in compressive deformation can be employed to reveal the evolution of flow mechanism.

In this paper, the compressive strain hardening exponents of the PS / LCS bimetal were calculated using the Hollomon's equation based on the isothermal compression tests. The effects of processing parameters including temperature, strain rate and plastic strain on the SHE were analysed in conjunction with the discussion of dynamic competition between the strain hardening and flow softening.

\section{Isothermal Compression Test}

As-received bimetal tube with $\sim 6 \mathrm{~mm}$ of LCS as the outer layer and $\sim 18 \mathrm{~mm}$ of PS as the inner layer was fabricated by centrifugal composite casting [1]. The chemical compositions of the LCS and PS are listed in Table 1. To perform the isothermal compression test on this bimetal, the cylindrical specimens, which are $10 \mathrm{~mm}$ in diameter and $20 \mathrm{~mm}$ in height, were machined from the same bimetallic billet. The bonding interface is perpendicular to the axis and the thickness of the LCS and PS layers are $\sim 5$ and $\sim 15 \mathrm{~mm}$, respectively.

Table 1 Chemical compositions of the LCS and PS (wt. \%)

\begin{tabular}{ccccccccc}
\hline Steel & $\mathrm{C}$ & $\mathrm{Si}$ & $\mathrm{Mn}$ & $\mathrm{P}$ & $\mathrm{S}$ & $\mathrm{Cr}$ & $\mathrm{Ni}$ & $\mathrm{Cu}$ \\
\hline LCS & 0.63 & 0.60 & 0.31 & 0.031 & 0.016 & 0.011 & 0.01 & 0.012 \\
PS & 0.12 & 0.22 & 0.37 & 0.023 & 0.015 & 0.011 & 0.01 & 0.009 \\
\hline
\end{tabular}

A serial of isothermal compression tests were carried out at a Gleeble 3500 thermomechanical simulator in the deformation temperatures ranging from 1073 to $1373 \mathrm{~K}$ at an interval of $100 \mathrm{~K}$, the strain rates of $0.02,0.1,1$ and $10 \mathrm{~s}^{-1}$, and the height reduction of $60 \%$. In order to minimise the bulging phenomenon, a tantalum foil combined with a graphite foil was placed between the anvil and the specimen to reduce the friction coefficient prior to test. The specimens were heated up to the deformation temperature with a heating rate of $20 \mathrm{~K} / \mathrm{s}$, and then held for $60 \mathrm{~s}$ for homogenisation. During the isothermal compression, the flow stress-strain curves, which are the essential data in computation of the SHE, were recorded automatically by the microprocessor of Gleeble 3500. After compressive deformation, the specimens were cooled in air to room temperature.

\section{Determination of Strain-hardening Exponent}

As is well established, the correlation between the true stress and true strain in the uniform plastic deformation stage can be expressed by the Hollomon's equation [9] as Eq. (1) when the temperature and strain rate remain unchanged.

$$
\sigma=K \varepsilon^{n}
$$

where, $\sigma$ is the true stress (MPa), $K$ is the strength coefficient (MPa), $\varepsilon$ is the true strain, and $n$ is the SHE. Taking the logarithm of both sides of Eq. (1), the Hollomon relation is represented by means of Eq. (2) as follows,

$$
L n \sigma=L n K+n L n \varepsilon
$$

By differentiating Eq. (2) leads to,

$$
n=\left[\frac{d \operatorname{Ln} \sigma}{d \operatorname{Ln} \varepsilon}\right]_{\dot{s}_{2} T}
$$

Thus, the $n$ value can be calculated as the instantaneous slope of a point in the double logarithmic true stress-strain plot. 


\section{Results and Discussion}

Fig. 1 shows the $\operatorname{Ln} \sigma-\operatorname{Ln} \varepsilon$ curves for the PS / LCS bimetal deformed at various strain rates and temperatures. It was found that the logarithmic relationship between the flow stress and plastic strain is highly non-linear, which is different from that obtained in uniform tensile deformation [6]. This non-linear feature indicates that the value of the SHE is a function of plastic strain during hot compression at a given temperature and strain rate.
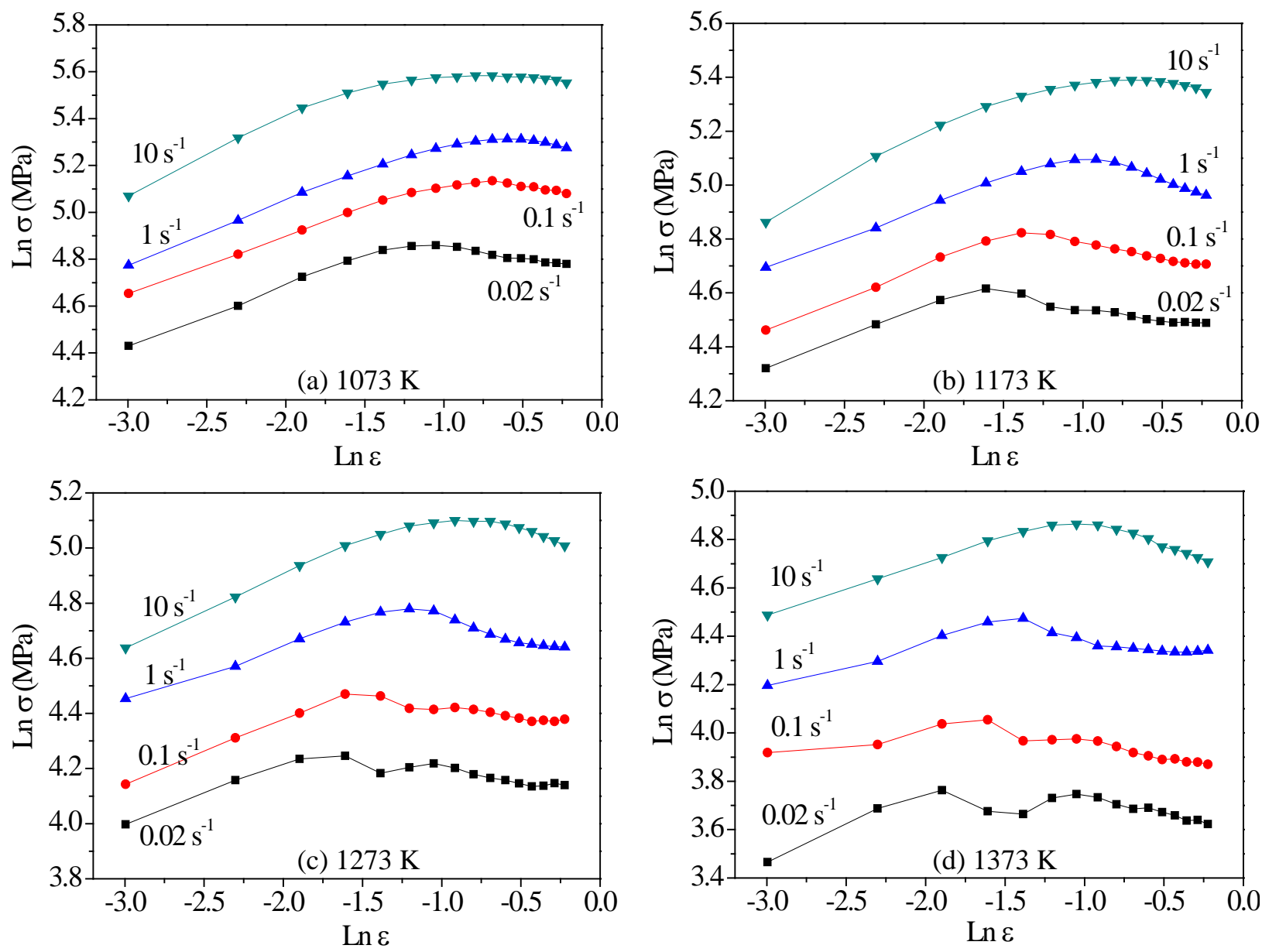

Fig. 1 The double logarithmic true stress-strain curves of the PS / LCS bimetal subjected to different strain rates and temperatures

Although the curve level increases with a decrease of temperature and an increase of strain rate, the variation of the SHE at a given plastic strain was not revealed clearly in Fig. 1. However, the varying patterns of the instantaneous slope along the curves imply that the dynamic competition between the strain hardening and dynamic softening. For all the testing cases, $\operatorname{Ln} \sigma$ increase with increasing $\operatorname{Ln} \varepsilon$ until a peak value was reached. The value of the SHE in this stage is positive as shown in Fig. 2, which means that the strain hardening is in the ascendant during the competition. Once the $\operatorname{Ln} \sigma$ over the first peak, the softening regimes like dynamic recovery (DRV) and dynamic recrystallisation (RDX) predominate the deformation so that the SHE is down to negative. The balance turns to the softening because of the dislocation annihilation due to the DRV or DRX [10,11]. Furthermore, it is noted that both increasing strain rate and decreasing temperature can enhance the strain hardening, which results in the positive SHE extending to a higher plastic strain.

For the strain rates of 1 and $10 \mathrm{~s}^{-1}$, the $\operatorname{Ln} \sigma-\operatorname{Ln} \varepsilon$ curves show the single peak pattern in Fig. 1. While the corresponding SHE profiles in Fig. 2 not only illustrate the superiority of dynamic softening by negative SHE value, but also imply the tendency of secondary strain hardening by an increase of the SHE through a valley. The secondary strain hardening behaviour was more significant with decreasing strain rate and increasing temperature, even though the DRX was the dominant player in 
this stage because the same compression condition is more beneficial to microstructural reconstitution in a longer accommodation time.
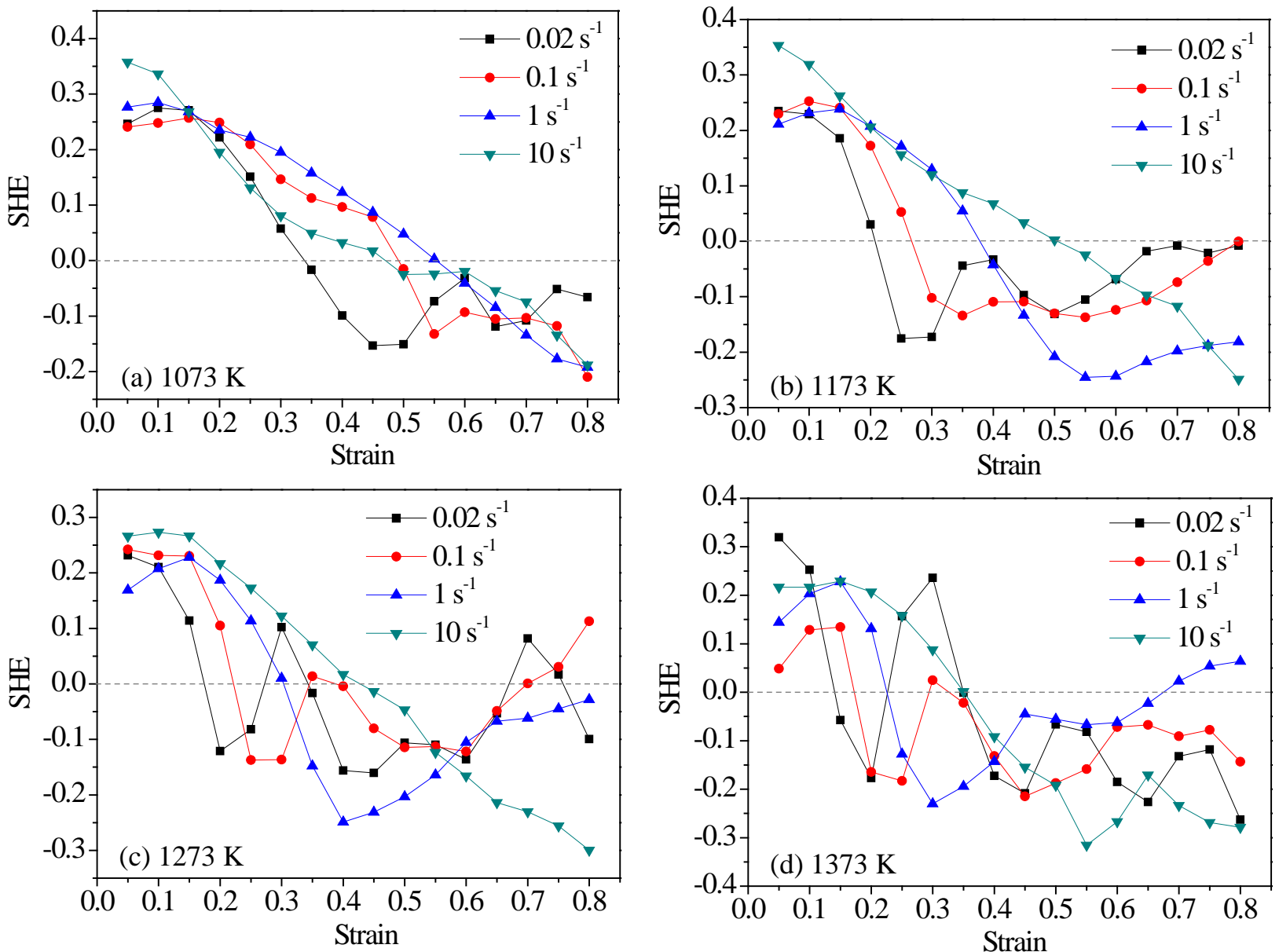

Fig. 2 The effect of strain on the SHE during hot compression of the PS / LCS bimetal at different strain rates and temperatures
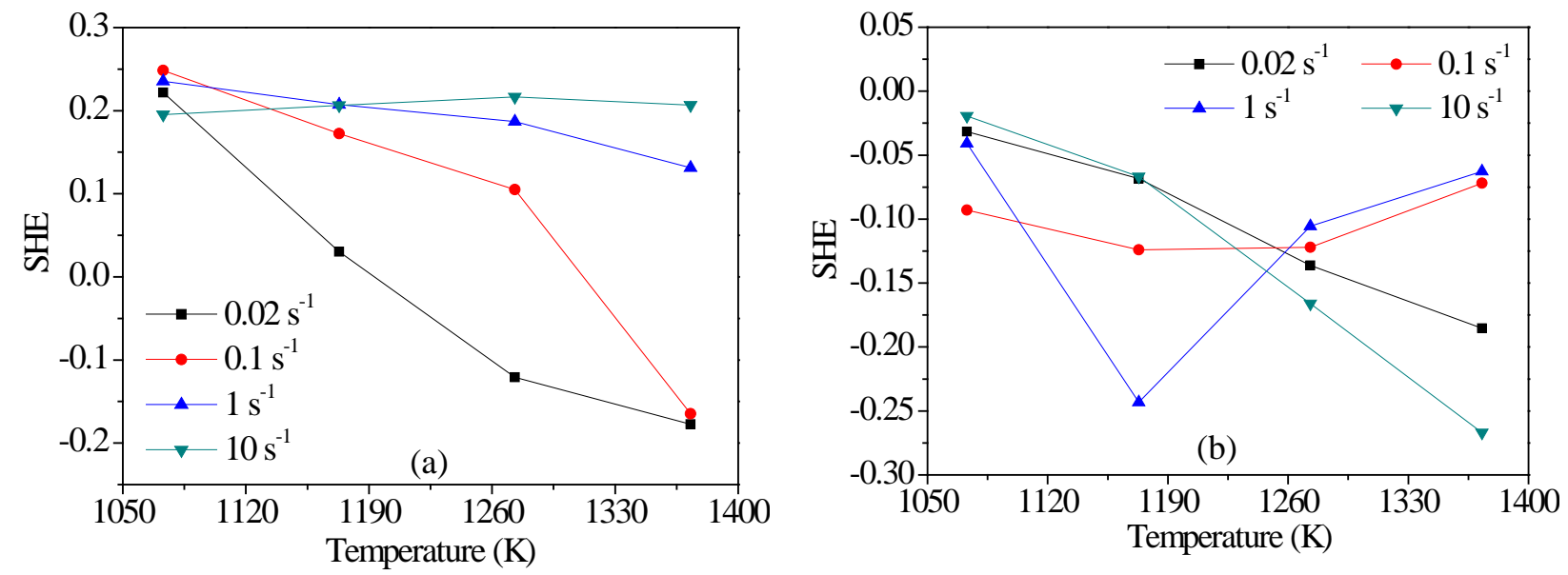

Fig. 3 The effect of temperature on the SHE during hot compression of the PS / LCS bimetal at true strains of 0.2 (a) and 0.6 (b)

Fig. 3 shows the influence of deformation temperature on the SHE during the isothermal compression of the bimetal. When the true strain is 0.2, as can be seen in Fig. 3(a), the value of the SHE changes slightly with an increase of temperature at a higher strain rate, i.e. 1 and $10 \mathrm{~s}^{-1}$. While a monotonic decrease of the SHE was observed by lowering the strain rate to 0.02 and $0.1 \mathrm{~s}^{-1}$. This difference indicates that the critical plastic strain for activating the softening regime at higher 
temperature combined with smaller strain rate is less than that at a higher strain rate. When the true strain is 0.6, all of the SHE values are negative as shown in Fig. 3(b), even though the plots are oscillatory at strain rates of 0.1 and $1 \mathrm{~s}^{-1}$.

\section{Conclusions}

(1) The non-linear $\operatorname{Ln} \sigma-\operatorname{Ln} \varepsilon$ curves of the PS / LCS bimetal during the isothermal compression process manifest that the compressive SHE was significantly affected by deformation temperature, strain rate and plastic strain.

(2) Although there are some fluctuations in the SHE - strain plots, the value of the SHE decreases roughly with increasing plastic strain. The competition between strain hardening and dynamic softening was presented by varying the SHE in the range of 0.4 to -0.4 .

(3) The variation of the SHE with true strain is dependent on the strain rate and temperature. The secondary strain hardening behaviour was revealed by the presence of multi-peaks in the SHE - strain profiles when the strain rate was decreased and the temperature was increased.

(4) In the early stage of the isothermal compression, a higher strain rate combined with a lower temperature contributes to enhance strain hardening, while the reverse condition reduces the critical plastic strain for activating dynamic softening of the bimetal.

\section{Acknowledgements}

The authors appreciate Mr. Bob de Jong and Dr. Liang Chen for their kind assistance and help during hot compression tests. The first author also thank the China Scholarship Council (CSC) for supporting the current research work.

\section{References}

[1] X.J. Gao, Z.Y. Jiang, D.B. Wei, H.J. Li, S.H. Jiao and J.T. Han, in: The Fifth Baosteel Biannual Academic Conference, Shanghai, China, 2013, pp. C110-116.

[2] X.J. Gao, Z.Y. Jiang, D.B. Wei, H.J. Li, S.H. Jiao and J.T. Han: submitted to Journal of Materials \& Design (2013).

[3] X.J. Gao, Z.Y. Jiang, D.B. Wei, H.J. Li, S.H. Jiao and J.T. Han: submitted to Journal of Materials Science and Engineering, A (2013).

[4] H. Mecking and U.F. Kocks: Acta Mater. Vol. 29 (1981), p. 1865.

[5] H.S. Chen, J.J. Gilman and A.K. Head: J. Appl. Phys. Vol. 35 (1964), p. 2502.

[6] S. Nagarjuna, B. Gopalakrishna and M. Srinivas: Mat. Sci. Egn., A Vol. 429 (2006), p. 169.

[7] J. Luo and M.Q. Li: Mat. Sci. Egn., A Vol. 538 (2012), p. 156.

[8] J. Luo, M.Q. Li, W.X. Yu and H. Li: Mater. Des. Vol. 31 (2010), p. 741.

[9] J.H. Hollomon: Trans. AIME Vol. 162 (1945), p. 268.

[10] H. Beladi, P. Cizek and P.D. Hodgson: Scripta Mater. Vol. 62 (2010), p. 191.

[11] E.I. Galindo-Nava, J. Sietsma, P.E.J. Rivera-Diaz-del-Castillo: Acta Mater. Vol. 60 (2012), p. 2615. 